\title{
Laryngeal elevation for endoscopic submucosal dissection in cervical superficial esophageal cancer at esophageal entrance
}

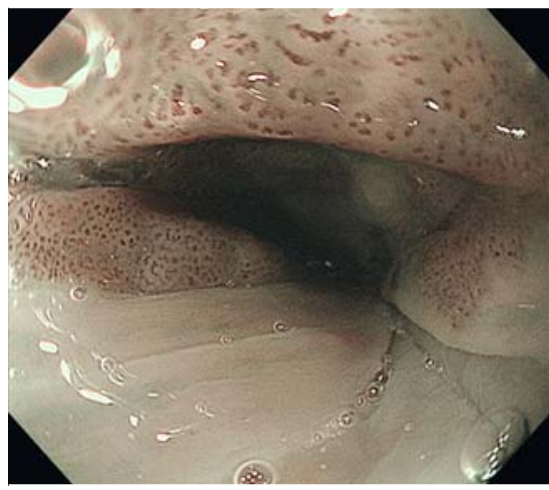

- Fig. 1 Endoscopic image with narrowband imaging showing a cervical superficial esophageal cancer during endoscopic examination using pethidine hydrochloride. Upper sphincter contraction and the pharyngeal reflex presented a challenge in capturing the entire lesion.

Endoscopic submucosal dissection (ESD) for cervical superficial esophageal cancer (CSEC) is an effective and safe procedure $[1,2]$. However, in CSEC extending to the esophageal entrance, ESD is technically challenging because of upper sphincter muscle tightening, a narrow working space, and the pharyngeal reflex. General anesthesia relaxes the sphincter muscle and aids endoscope maneuverability. Laryngeal elevation using a laryngoscope facilitates a wider working space and is generally used in ESD for pharyngeal superficial cancer [3], but has not previously been reported in ESD for CSEC.

A 59-year-old man diagnosed with a 40-mm, half-circumferential superficial squamous carcinoma at the cervical esophagus, which extended to the esophageal entrance, was referred to our hospital. During endoscopic examination using pethidine hydrochloride [4], sphincter contraction and pharyngeal reflex meant that it was difficult to capture the entire lesion ( $>$ Fig.1). ESD under general anesthesia with endotracheal intubation and laryngeal elevation was therefore performed. The otolaryngologist elevated the larynx using a Sato's curved laryngo-

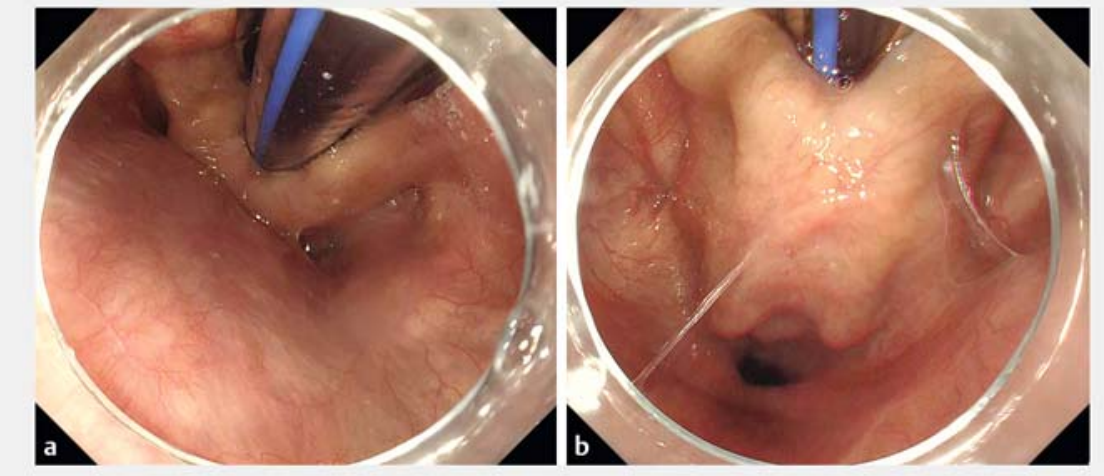

- Fig. 2 a By pulling the laryngoscope up behind the intubation tube, laryngeal elevation was achieved. b Good visibility of the esophageal entrance resulted.

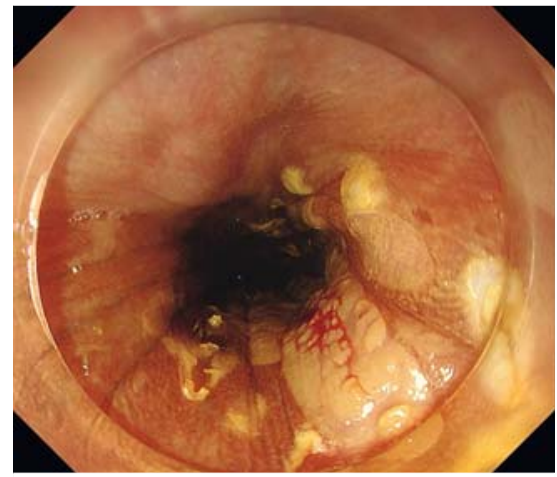

Fig. 3 Endoscopic images with iodine staining of the lesion surrounded by marking dots. Laryngeal elevation allowed the entire lesion to be visualized.

scope (Nagashima Medical Instruments Co., Ltd., Tokyo, Japan), which widened the esophageal entrance, enabled observation of the entire lesion, and improved endoscope maneuverability ( $\mathbf{F i g . 2}$, - Fig.3). Resection using a Flushknife-BT (1.5 mm, DK2618JB; Fujifilm Medical, Tokyo, Japan) and transparent hood (D-201-11804; Olympus Corporation, Tokyo, Japan) was started from the proximal side of the lesion. Using traction-assisted ESD [5], en bloc resection was achieved within 45 min without adverse events (> Fig.4; > Video 1). Histopatho-

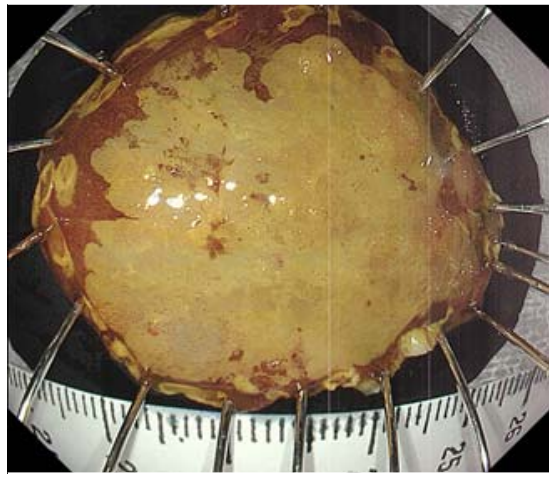

- Fig. 4 Macroscopic appearance of the resected specimen.

logical analysis of the specimen showed mucosal cancer with negative margins.

In this case, laryngeal elevation using a laryngoscope was employed to widen the esophageal entrance and provide a favorable working space, leading to successful ESD. We have experience of two further cases of CSEC extending to the esophageal entrance in which successful ESD was achieved by laryngeal elevation. In conclusion, laryngeal elevation represents an effective and safe component of ESD for CSEC extending to the esophageal entrance.

Endoscopy_UCTN_Code_TTT_1AO_2AG 


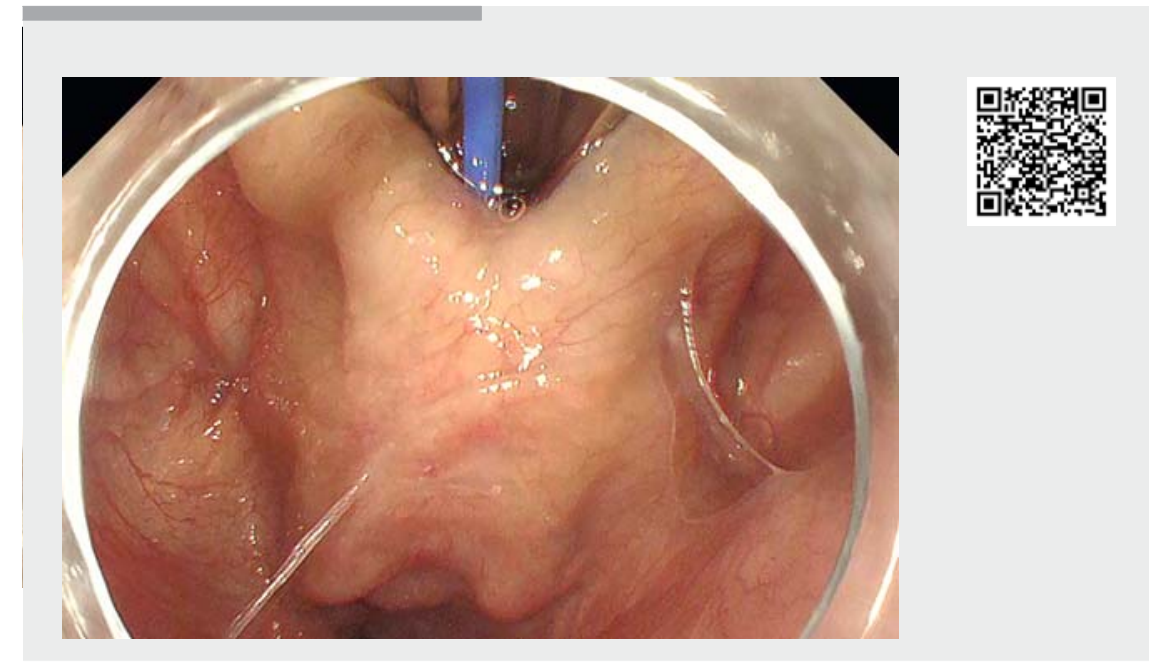

Video 1 Laryngeal elevation provided a favorable working space at the esophageal entrance, which led to successful ESD.

\section{Acknowledgment}

We thank Clare Cox, PhD, from Edanz Group, for editing a draft of this manuscript.

\section{Competing interests}

The authors declare that they have no conflict of interest.

The authors

Takahiro Inoue ${ }^{1}$, Satoki Shichijo ${ }^{1}$, Hironori $\mathrm{Cho}^{2}$, Ayaka Shoji ${ }^{1}$, Kotaro Waki ${ }^{1}$, Takashi Fujii' ${ }^{2}$, Ryu Ishihara ${ }^{1}$

1 Department of Gastrointestinal Oncology, Osaka International Cancer Institute, Osaka, Japan

2 Department of Head and Neck Surgery, Osaka International Cancer Institute, Osaka, Japan
[3] Hanaoka N, Ishihara R, Takeuchi Y et al. Clinical outcomes of endoscopic mucosal resection and endoscopic submucosal dissection as a transoral treatment for superficial pharyngeal cancer. Head Neck 2013; 35: 1248-1254

[4] Yamasaki Y, Ishihara R, Hanaoka $\mathrm{N}$ et al. Pethidine hydrochloride is a better sedation method for pharyngeal observation by transoral endoscopy compared with no sedation and midazolam. Dig Endosc 2017; 29: $39-48$

[5] Shichijo S, Takeuchi Y, Fukuda $\mathrm{H}$ et al. Whole-fornix endoscopic submucosal dissection for gastric mucosal adenocarcinoma. Endoscopy 2020. doi:10.1055/a-1085 9472

\section{Bibliography}

Endoscopy 2021; 53: E65-E66

DOI $10.1055 / \mathrm{a}-1187-0826$

ISSN 0013-726X

published online 19.6.2020

(c) 2020. Thieme. All rights reserved.

Georg Thieme Verlag KG, Rüdigerstraße 14, 70469 Stuttgart, Germany

\section{Satoki Shichijo, MD, PhD}

Department of Gastrointestinal Oncology, Osaka International Cancer Institute, 3-1-69, Otemae, Chuo-ku, Osaka, 541-8567, Japan Fax: +81-6-69451900

shichijiyou-tky@umin.ac.jp

\section{References}

[1] lizuka T, Kikuchi D, Hoteya S et al. Efficacy and safety of endoscopic submucosal dissection for superficial cancer of the cervical esophagus. Endosc Int Open 2017; 5: E736E741

[2] Ariyoshi R, Toyonaga T, Tanaka S et al. Clinical outcomes of endoscopic submucosal dissection for superficial esophageal neoplasms extending to the cervical esophagus. Endoscopy 2018; 50: 613-617

\section{ENDOSCOPY E-VIDEOS}

https:/|eref.thieme.de/e-videos

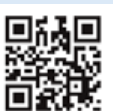

Endoscopy E-Videos is a free access online section, reporting on interesting cases and new

techniques in gastroenterological endoscopy. All papers include a high quality video and all contributions are freely accessible online.

This section has its own submission website at https://mc.manuscriptcentral.com/e-videos 